\title{
Estimating a new approach for describing electrical conductivity parameters in partially saturated sediments
}

\author{
F. Ali Swaid \\ The Great Man-made River Water Utilization Authority, Phase One, \\ Sirt, Libya
}

\begin{abstract}
Electrical resistivity imaging has the capacity to map changes in soil water content and quality in the vadose zone. However, interpretation of the images is difficult because of the numerous variables that influence the measured resistivity. The dominant variables include porosity, degree of saturation, pore water salinity, clay content and clay type.

There have been numerous models published that combine these variables in order to predict the bulk electrical conductivity of any mixture. All these equations need to be calibrated against controlled samples in order to determine parameters that account for geometrical effects and grain surface conduction.

Recently, Glover et al. (Earth and Planetary Science Letters 2000) published an extension to Archie's equation to account for the nonlinear behavior seen in the relationship between pore water salinity and soil/rock bulk conductivity. In this paper the Glover et al. model is extended to partial saturation conditions.

The application of the new model in the evaluation of salinity and changes of soil moisture is possible wherever the required criteria are met. Furthermore, more, this relation can also be taken to estimate the changes in soil moisture caused by root uptake.
\end{abstract}

Keywords: partial saturation, cation exchange capacity, saturation exponent, geometric factor, water conductivity, bulk conductivity.

\section{Introduction}

Soil scientists routinely map soil salinity in agricultural area using electromagnetic induction surveys (Chang et al. [4]; Rhoades et al. [31]). But 
electrical conductivity measurements also have the potential to map variation in moisture content and clay content.

The application of electrical methods for measuring soil and rock properties including water content, cation exchange capacity (CEC), salinity and clay content dates from early in the $20^{\text {th }}$ century for the petroleum industry (Archie [2]; Smith-Rose [36]; Wenner [43]). The determination of salinity through the measurement of electrical conductance has been well established for decades (U.S. Salinity Laboratory Staff, 1954). It is known that the electrical conductivity of water is a function of its chemical composition (McNeal et al. [24]). Soil salinity investigation has become widespread since the 1970s (Rhoades et al. [26]); Rhoades and Van Schilfgaarde [27]; and reviewed lately by Friedman [13]. In the last two decades, electrical measurements have become the main way to characterize, map and monitor soil properties such as pore water (salinity), clay and moisture content. Due to improvements in the technology, soil electrical conductivity measurements are now being made at the field scale using both direct contact sensors (e.g. direct current, DC) to measure resistance and noncontact sensors based upon electromagnetic induction technology (Dolittle et al. [11]; Jaynes et al. [18]). The two approaches provide highly correlated measures of apparent electrical conductivity of soil that show correlation with soil salinity, CEC and soil moisture in the vadose zone (Acworth et al. [1]; Clavier et al. [5]; Lund et al. [23]).

The electrical conductivity of sediment depends on different parameters such as the spatial distribution of the constituent minerals and pore space and saturation distribution.

This paper reviews the information on the phenomenon of electrical conductivity and its specific applications for mapping and monitoring soil salinity. A new electrical conductivity model is developed that can address the value of the saturation exponent $(n)$ in partially saturated soil. The new model offers a better interpretation of the electrical conductivity to represent salinity and moisture in soil.

\section{Concept of electrical conductivity measurement}

Electrical resistivity is averaged measurement of the electrical resistivity of a material under an applied electrical field. The resistivity of a block of material in defined as:

$$
\rho=R(A / L)
$$

where $\mathrm{L}$ is the length of the block (m), A is the cross-sectional area $\left(\mathrm{m}^{2}\right)$ and $\mathrm{R}$ is the electrical resistivity $(\mathrm{ohm})$. Thus, the units of resistivity are ohm.m.

The reciprocal of resistivity is conductivity $(\sigma)$, where $\sigma=(1 / \rho)$. The units of measurement of conductivity are $\mathrm{S} / \mathrm{m}$, but soil measurements are typically reported in $\mathrm{dS} / \mathrm{cm}$ and water conductivity measurements in $\mu S / \mathrm{cm}$. 


\subsection{Soil electrical conductivity (EC)}

An electrical current can be transferred by the flow of free electrons in a macropore, along the surfaces of soil minerals (i.e. exchangeable ions), and through alternating layers of particles and solution (Rhoades et al. [31]). Conduction via the exchangeable cations that reside near the surfaces of charged fine soil particles affect conductivity since the cations are electrically mobile to various extents within the double layer of the soil particle (Nadler and Frenkel [25]). There are other factors that have secondary effect on the electrical conductivity like soil temperature and pressure, which will not be discussed here.

\subsection{Total dissolved solids}

Total Dissolved Salt (TDS) is a measure of the total ions (e.g. calcium, magnesium, sodium and bicarbonate) in solution. Water electrical conductivity $\left(\sigma_{w}\right)$ is actually a measure of the ionic activity of a solution in term of its capacity to transmit current. In a dilute solution $T D S$ and $\sigma_{w}$ are reasonably comparable and the TDS of a water sample based on the measured EC value can be calculated using equation 2 . However it is important to note that the main factor which affects the electrical conductivity of the soil solution is the water content (Friedman [13]).

$$
\sigma_{w}=(T D S / C)
$$

where $\sigma_{w}$ is water electrical conductivity $(\mu \mathrm{S} / \mathrm{cm}), T D S$ is total dissolved salts $(\mathrm{mg} / \mathrm{l})$, and $C$ is constant of proportionality which ranges from 0.54 to 0.96 (Hem [15]) according to the chemistry of the water. This relationship takes into account the volume of anions and cations (dissolved salts) content within the soil solution, which is also used as a soil salinity indicator.

\subsection{Origin of particle charge}

Surface charge on soil colloids is developed in two ways:

1. By isomorphic substitution which is the replacement of one atom by another of similar size in a crystal lattice without disrupting or changing the crystal structure of the mineral, (Pfannkuch [27]). A negative charge of the anions is balanced by the positive charge of the cations that are coordinated to it. Net negative charge is developed when a cation of similar size and less positive charge substitutes for one of higher positive charge (e.g. $\mathrm{Al}^{3+}$ by $\mathrm{Mg}^{2+}$ or $\mathrm{Ca}^{2+}$ and $\mathrm{Mg}^{2+}$ or $\mathrm{Ca}^{2+}$ by $\mathrm{Na}^{1+}$ or $\mathrm{K}^{1+}$ ), (Lindsay [21]). Isomorphic substitution can also take place between cations of the same charge or a cation of higher positive charge. In the case of isomorphic substitution between cations of the same charge, no charge is developed while, in the case of isomorphic substitution between a cation of higher positive charge with one of lower positive charge a net positive charge is developed.

2. By the dissociation of $\mathrm{H}^{+}$from active molecules located at the border of the exchange complex, creating negative sites, or to the protonation into $\mathrm{OH}_{2}{ }^{+}$, 
giving positive charges. Protons $\left(\mathrm{H}^{+}\right)$may for example be released by acid groups at the broken edges of clay particles, or by carboxyl or phenol groups in the organic material, or by aluminum and iron hydroxides. The dissociation of $\mathrm{H}^{+}$creates vacancies, which may be filled by metallic ions. It is strongest at high concentrations of $\mathrm{OH}^{-}$in the soil solution and is therefore called the $\mathrm{pH}-$ dependent part of the cation exchange capacity which increases with rising $\mathrm{pH}$. At low $\mathrm{pH}$ values this type of cation exchange capacity may completely disappear, (Stewart and Hossner [37]).

A clay particle attracts cations to its surface from its surroundings as it tries to keep electrical neutrality (Vezenov et al. [41]). Usually these cations are not part of the crystalline structure of the clay; they are simply drawn from the aqueous solution that surrounds the clay. They may be replaced by other ions when the clay formation evolves and the aqueous environment changes. The quantity that is available for exchange depends on the number of negative charges present in the actual clay lattice, which is known as Cation Exchange Capacity (CEC). Ions held adjacent to the exposed lattice structure of the surface can be released into solution and ions in the solution can be drawn to sites on the surface. Clays can thus take ions from the solution and replace them with different ions from their structure (Lockhart [22]).

An important net effect is that when water is added to clay the concentration of ions is increased in the solution in the vicinity of clay surface. On the other hand when the sediment is dry the counterions are fixed to the surface (Clavier et al. [5]).

\subsection{Surface conduction}

The electrical conductivity of sediment is obtained from the conduction through the bulk solution occupying the pores and from surface conduction occurring at the water/grain interface. Surface conduction is characterized by the specific surface conductance which is the sum of three components (Revil and Glover [29]): Conduction within the electrical diffuse layer, which makes a negligible contribution to the total specific surface conductance;

i. Conduction in the Stern layer, which varies significantly with the salinity of the pore fluid at low salinities;

ii. A mechanism operating directly on the mineral surface, independent of salinity, and perhaps associated with proton transfer.

The net negative charge generated by clay particles as discussed in the previous section will be balanced or neutralized by adsorbing cations. Therefore, some of this negative charge will be used to exclude anions from the area adjacent to the negatively charged clay surface. This repulsion is often called negative adsorption. There will be a greater concentration of cations adjacent to the clay surface than in an area further away from the greater concentration of anions. Eventually, the distribution of cations and anions will be the same as the bulk solution. The combination of the negatively charged clay surface and unequal distribution of cations and anions (compared to the bulk solution) adjacent to the clay particle is called the electrical double layer or diffuse double layer (Quirk [28]). 
The electrical double layer is composed of a fixed layer (Stern layer) and a diffuse layer (Gouy layer). In the Stern layer, the ions are assumed to oscillate about fixed adsorption sites, whereas, in the diffuse layer, ions are assumed to undergo Brownian motion. In a porous plug of clay, the surface becomes negatively charged when wetted with water. The adjoining Stern layer of liquid, which carries positively charged ions, balances this charge. The thickness of the Stern layer is approximately the radius of a hydrated cation adsorbed on the clay particle surface, (Clavier et al. [5]). Three planes divide the Stern layer and the diffuse layer: one is the plane of the clay-water interface; a second is the outer Helmholtz plane (O.H.P); and the third is the plane of shear.

The diffuse layer has a higher conductivity than the normal pore fluid and represents a parallel low resistivity path for current conduction along the mineral surfaces (Schmitz [34]). Clay particles like montmorillonite and nontronite have an enormous surface area per unit weight. Therefore, a relatively small amount of clay can dramatically increase the bulk conductivity. The effect is most dramatic when the pore water is of high resistivity and is insignificant for highly concentrated pore water, which carries the current easily without the benefit of the surface conduction paths.

\section{Models of bulk electrical conductivity}

Predicting the electrical properties of sediments is an intractable problem, and there have been numerous models proposed to predict the bulk electrical properties of sediments based on the constituents of the soil or rock. Many of these models have been reviewed by Worthington [47]; Glover et al. [14]; and Friedman [13]. Early measurements of the electrical properties of rocks were primarily conducted by Wenner [43]; Smith-Rose [36]; Archie [2]; Winsauer et al. [44]; Hill and Milburn [16]. This was followed by several improvements in the models by many geophysicists, for example Waxman and Smits [42]; Pfannkuch [27]; Rhoades and Van Schilfgaarde [33]; Bussian [3]; Kelly [20]; Silva and Bassiouni [35]; Rhoades et al. [31]; Worthington and Pallat [47]; de Lima and Sharma [7]; de Lima and Niwas [9]; Toumelin and Torres-Verdin [39]. These models have improved the knowledge in the area of petroleum investigation. The majority of these models are variants of Archie's law [2]. These models range from empirical equations to equations that are developed from the best fit of experimental data. The major electrical conductivity models are reviewed below.

\subsection{Clay free, high salinity sediments - Archie's Law [2]}

Archie [2] showed that the conductivity of a rock depends on the porosity, particle shape and size distribution and the direction of measurements. Archie defined the formation factor as the ratio of the conductivity of the pore water, which saturates a particulate medium consisting of non-conductive particles, to the conductivity of the mixture of particles and pore fluid. The formation factor, $F$ is given by: 


$$
(1 / F)=\left(\sigma_{b} / \sigma_{w}\right)
$$

where $\sigma_{w}$ is the conductivity of the pore water and $\sigma_{b}$ is the bulk conductivity of the soil or rock.

Archie's Law relates $F$ to porosity $(\phi)$ :

$$
\sigma_{b}=\sigma_{w} \phi^{m}
$$

where $\phi$ is the porosity, and $m$ is the geometric factor (historically called the cementation factor) and the exponent $m$ depends on grain shape consolidation, texture, and cementation. Archie found $m$ values between 1.8 and 2 for consolidated sandstones and near 1.3 for sands. Jackson et al. [17] studied uncompacted natural and artificial sandstones for which $m$ increases from 1.2 to 1.9 for particles with decreasing sphericity. No relationship with the particle sizes or the size distribution of particles was found.

Equation (4) was extended by Winsauer et al. [44] as an empirical formula for various sandstone formations:

$$
\sigma_{b}=a \sigma_{w} \phi^{m}
$$

where $a$ is an empirical constant introduced to improve the line of best fit to experimental data. The constant $a$, however, has no physical meaning and the limit $\phi$ tends to one. The literature reports values for $m$ being calibrated using equation (5). Friedman [13] has tabulated measurements of $m$ in the literature and the reported ranges varying from 1.2 for glass spheres to 4.4 for altered tuff.

\subsection{Low pore water salinity and clay bearing sediments}

Earlier studies of Archie's Law recognized that other factors might affect conductivity, which are not included in the formulation mentioned above. The most significant of these other factors is the clay content of a rock (Keller [19]; Patnode and Wyllie [26]). Rocks, which contain significant amounts of clay, tend to have higher conductivities than predicted by the use of Archie's Law. Some researchers treat the clay minerals as being electrically conductive and connected more or less in parallel with electrolyte filled pore structures, (de Lima et al. [8]). Other investigators treat clay as a substance which electrolyses in the same manner as salt, but which is non-conductive in the dry state. In either case in clay-rich rocks, some provision must be made for the added conductance representing clay minerals.

Equation (5) is applicable only to clean or clay mineral free sands (nonconductive phase). When clay minerals are present, there is a negative charge excess near the clay mineral surface. Electrical neutrality requires hydrated cations from the pore electrolyte. These hydrated cations on the surface of the clay minerals are in rapid exchange with those in the pore electrolyte.

It has been well established in the presence of clay that a non-linear model is needed to account for the initial upward inflection observed on linear scaled $\sigma_{b}$ versus $\sigma_{w}$ graphs (Winsauer and McCardell [45]; Hill and Milburn [16]; 
Waxman and Smits [42]; Nadler and Frekel [25]; Rhoades et al. [31]; de Lima et al. [8]). The dominant models in the literature are reviewed below.

\subsection{Waxman and Smits [42] model}

They based their model on assumptions as follows:

The rock can be represented by two parallel resistances. One represents the pore fluid, the other with the double layer (clay minerals present with the rock). The double layer conductivity depends on the electrolyte conductivity. It is the result of CEC at the clay surface and proportional to cation concentration $\left(Q_{v}\right)$.

They have attempted to define the relationship, in a mechanistic way between clay content and electrical conductivity response of shaley sand. According to their model a clayey formation behaves like a clean formation of the same porosity, tortuosity, and fluid saturation except that the water appears to be more conductive than expected from its bulk salinity. The excess conductivity is attributed to the presence of the exchange cations held on the clay surface.

Taylor and Barker [38] noted that, the Waxman and Smits model incorrectly assumes the exchange cations are distributed evenly throughout the electrolyte, rather than occurring at the clay surfaces. It also assumes that, the matrix conductivity is dependent on fluid conductivity for which there is no theoretical justification. The basic relationship is:

$$
\sigma_{b}=(1 / F)\left(\sigma_{w}+B Q_{v}\right)
$$

where $\sigma_{b}$ is the bulk conductivity, $\sigma_{w}$ is the fluid electrical conductivity, $B$ is the counter ion equivalent conductance, which describes the average mobility of the ions, and $Q_{v}$ is the counter ion charge concentration (meq/m), which is directly related to the cation exchange capacity.

\subsection{Clavier et al. [6] model}

Some effects relate to the adsorptive properties of the clays that had not been taken by the Waxman and Smits model into account, namely clay water that is a result of the double layer associated with the clay. Clavier et al. [6] have attempted to suppress this contradiction by using a their model. The Double layer is assumed to contain mainly positive charges, which balance the negative charges on the clay surface. This diffusion layer can be considered as a salt free zone and its effect continues up to some distance from the clay surface. Hence, the pore space of clayey sand is assumed to be filled with the clay water and far water. Each one of these waters occupies a fraction of the available pore space that are called clay water porosity and far water porosity.

The Clavier et al. and Waxman Smits model consider that the conductivity of the saturating fluid is complemented by the conductivity of clay counterions. The basic difference between the Clavier et al. model and Waxman and Smits model is that the Clavier et al. model considers both the far water and the clay water with specific conductive properties. Also, the Clavier et al., model characterizes 
the rock formation by conductivity of clay water formation factor $F$, shaliness parameter $\mathrm{Q}_{\mathrm{v}}$, and its bulk conductivity $\sigma_{b}$ observed at fluid conductivity.

$$
\sigma_{b}=(1 / F)\left\lfloor\left(1-v_{Q} Q_{v} \sigma_{w}\right)+v_{Q} Q_{v} \sigma_{c w}\right\rfloor
$$

where $\sigma_{c w}$ is conductivity of clay water (double layer) and $v_{Q_{v}}$ is the amount of clay water associated with 1 unit (meq) of clay counterions.

\subsection{Bussian's model [2]}

This model allows for different paths of conduction. It considers a granular rock composed of homogeneous conductive particles having conductivity $\sigma_{r}$ embedded in a conductive host fluid of conductivity $\sigma_{w}$. The surface conductivities of the clay platelets can be represented by equivalent volume conductivity $\sigma_{r}$. The clay platelets are treated as bulk conductivity $\sigma_{b}$. The model has been used to relate the electrical properties of a heterogeneous mixture to the properties of individual components.

$$
\sigma_{b}=\sigma_{w} \phi^{m}\left(\left(1-\left(\sigma_{r} / \sigma_{w}\right)\right) /\left(1-\left(\sigma_{r} / \sigma_{b}\right)\right)\right)^{m}
$$

where $\sigma_{r}$ is grain soil conductivity or surface conduction. When $\sigma_{r} \rightarrow 0$, especially in sand $\left(\sigma_{w}>>\sigma_{r}\right)$ this leads to Archie's law for a single conducting medium (Equation (9)).

$$
\sigma_{b}=\sigma_{w} \phi^{m}
$$

\subsection{De Lima and Sharma [7]}

They used dual distributions of conductives in a formulation based on Bussian's model [3] to account for the effect dispersed and coating clay on electrical conduction. They have modified this model to an electrical conductivity equation that includes the ionic composition of the pore water and the electrical double layer.

$$
\sigma_{b}=(1 / F)\left(\sigma_{w}+m(F-1) \sigma_{r}\right.
$$

\subsection{Rhoades et al. model [31]}

Rhoades et al. [31] developed Archie's empirical model to relate soil volume and volume of water (Equation (11)) in which the relationship between the porosity and conductivity developed by Faust [12] was used.

$$
\left.\sigma_{b}=\left\{\left(\theta_{r}+\theta_{w r}\right)^{2} \sigma_{w r} \sigma_{r}\right] /\left[\theta_{r} \sigma_{w r}+\theta_{w s} \sigma_{r}\right]\right\}+\left(\theta_{w}-\theta_{w r}\right) \sigma_{w r}
$$

where $\theta_{r}$ is the volume of soil, $\theta_{w}$ is the volume of water, $\theta_{w r}$ is the volume of series coupled soil and water, $\theta_{w s}$ is the conductivity of the mobile pore water, 
$\sigma_{r}$ is the conductivity due to the soil particles, $\sigma_{w r}$ is the conductivity of immobile water and $\sigma_{b}$ is bulk sample conductivity.

Rhoades et al. [31] find a relationship between the electrical conductivity of saturated sediment $\left(\sigma_{\mathrm{b}}\right)$ and the electrical conductivity of saturated sediment $\left(\sigma_{\mathrm{w}}\right)$, at three levels of volumetric water content $\left(\theta_{w}\right)$. This relationship shows that the $\sigma_{\mathrm{b}}$ increases as $\theta_{w}$ increases and the $\sigma_{\mathrm{b}}$ and $\sigma_{\mathrm{w}}$ are proportional. However, according to Equation (10) the electrical conductivity of the soil is more dependent on the degree of saturation.

Glover et al. [14] (GHP) model

Another non-linear model is the modified Archie's law proposed by Glover et al. [14]. They approximate the bulk property of a medium as the summation of two phases.

$$
\sigma_{b}=\sigma_{w} \phi^{m}+\sigma_{r}(1-\phi)^{p}
$$

where $p$ represents the geometrical parameter of the conducting grains (conductivity of the grains). The exponent $p$ can be expressed in terms of the porosity and geometrical factor $m$ as:

$$
p=\left(\log \left(1-\phi^{m}\right)\right) /(\log (1-\phi))
$$

Combining equations (11) and (12) gives the approximation of the bulk conductivity as:

$$
\sigma_{b}=\sigma_{w} \phi^{m}+\sigma_{r}(1-\phi)^{\left(\left(\log \left(1-\phi^{m}\right) / \log (1-\phi)\right)\right.}
$$

This non-linear relationship is algebraically easier to handle compared to the other non-linear models. In equation (12) Archie's equation [2] is given in the limit as $\sigma_{r}$ tend to zero.

Archie's law and GHP model can be calibrated to data using the fit routine in gnuplot version 3.7 (http://www.gnuplot.info/). The fit algorithm is a non-linear least squares Marquardt-Levenberg algorithm.

\section{Partially saturated sediments}

Based on empirical observations of partially saturated rocks Archie [2] defined

$$
\rho_{P S}=I \rho_{b}
$$

where I is the resistivity index and $\rho_{P S}$ is the bulk resistivity of the partially saturated rock. Archie then noted that the resistivity index was related to the degree of saturated via:

$$
I=1 / S_{w}^{n}
$$

where $\mathrm{S}$ is the degree of saturation and $\mathrm{n}$ is the saturation exponent. Rearranging in terms of conductivity:

$$
\sigma_{P S}=S_{w}^{n} \sigma_{b}
$$


for samples of rock typically found in petroleum regions $n$ was found to be approximately equal to 2. Equation (17) is used below to extend the saturated GHP model to partial saturation.

\subsection{Extending the GHP model for partial saturation}

The GHP model is capable of simultaneously describing multiple soil property effects. It is hypothesized that it might be successfully used to represent the diverse variation in the observed electrical conductivity of partially saturated soil with surface conduction.

Substitution of equation (14) into equation (17), gives the GHP model for Partial Saturation, which will be called the $\left(\mathrm{GHP}_{\mathrm{PS}}\right)$ model, as follows:

$$
\sigma_{P S}=S_{w}^{n}\left(\sigma_{r}(1-\phi)^{\left(\left(\log \left(1-\phi^{m}\right) /(\log (1-\phi)\right.\right.}+\sigma_{w} \phi^{m}\right)
$$

where, $m$ is the volume fraction of soil, and $n$ is saturation exponent.

The GHP model considered that the bulk conductivity is a summation of pore water and the grains conductivity, in contrast the GHP $\mathrm{PS}_{\mathrm{PS}}$ consider the degree of saturation as a part of grains conductivity in partially saturation conditions.

\section{Conclusion}

In this paper, electrical conductivity models have been reviewed. These models were formulated to describe the electrical conductivity of sediments in the presence of clay and for saturated or unsaturated conditions. Most of these models were derived from Archie's law and they show limitations when used in different cases. These limitations are sedimentation related such as homogeneity and water wettability, which has one or two conducting phases. However, soil or rock is complex, and has different conditions in the case of partially saturated soil, which makes use of these models unstable.

The theory proposed by Glover et al. [14] to describe the electrical properties of a porous medium consisting of an insulating porous solid matrix for two conductive phases has been extended. The GHP $\mathrm{PS}_{\mathrm{PS}}$ model is derived to describe the partial conductivity of soil bearing clay. An expression is derived that relates the partial saturation to surface conduction, geometric factor, porosity and saturation exponent.

\section{References}

[1] Acworth, R.I., Young, R. R. \& Bernadi, A.L., Monitoring soil moisture status in a Black Vertosol on the Liverpool Plains, NSW, using a combination of neutron scattering and electrical image methods, Electrical image, black soils, lucerne Australian Journal of Soil Research, 43(2): 105-117, 2004.

[2] Archie, G.E., The electrical resistivity log as an aid in determining some reservoir characteristics. Transactions of the American Institute of Mining and Metallurgical Engineers, 146: 54-62, 1942. 
[3] Bussian, A.E., Electrical conductance in a porous medium. Geophysics, 48(9): 1258-1268, 1983.

[4] Chang, C., Sommerfeldt, T.G., Carefoo, J.M. \& Schaalje, G.B., Relationships of electrical conductivity with total dissolved salts and cation concentration of sulfate dominant soil extracts Canadian Journal of Soil Science, 63: 79-86, 1983.

[5] Clavier, C., Coates, G. \& Dumanoir, J., Theoretical and experimental bases for the dualwater model for interpretation of shaly sands. Society of Petroleum Engineers Journal, 24(2): 153-168, 1984.

[6] Clavier, C., Coates, G. \& Dumanoir, J. The theoretical and experimental bases for the, Dual Water, model for the interpretation of shaly sands. Society of Petroleum Engineers Journal, 6859: 16, 1977.

[7] de Lima, O.A.L. \& Sharma, M.M., A grain conductivity approach to shaly sandstones. Geophysics, 55: 1347-1356, 1990.

[8] de Lima, M., Ben, Clennell, Geraldo \& Sri. A volumetric approach for the resistivity response of freshwater shaly sandstones. Geophysics, 70(1), 2005.

[9] de Lima, O.A.L. \& Niwas, S., Estimation of hydraulic parameters of shaly sandstone aquifers from geoelectrical measurements. Hydrology, 235: 1226, 2000.

[10] de Lima, M., Ben, Clennell, Geraldo \& Sri, A volumetric approach for the resistivity response of freshwater shaly sandstones. geophysics, 70(1), 2005 .

[11] Dolittle, J., Ealy, E., Secrist, G., Rector, D. \& Crouch, M., Reconnaissance soil mapping of a small watershed using electromagnetic induction and global positioning system techniques. Soil Surv. Horiz, 36: 86-94, 1995.

[12] Faust, A Velocity Function Including Lithologic Variation. Geophysics, 18: 271-297, 1953.

[13] Friedman, S.P., Soil properties influencing apparent electrical conductivity: a review. Computers and Electronics in Agriculture, 46: 45$70,2005$.

[14] Glover, P.W.J., Hole, M.J. \& Pous, J., A modified Archie's law for two conducting phases. Earth and Planetary Science letters, 180: 369-383, 2000 .

[15] Hem, J.D., Study and Interpretation of the Chemical Characteristics of Natural Water, 3rd edition. U.S. Geological Survey, Washington, DC, . Water-Supply, 2254: 263, 1989.

[16] Hill, H.J. \& Milburn, J.D., Effect of clay and water salinity on electrochemical behavior of reservoir rocks. Trans. AIME 207:65-72, 1956.

[17] Jackson, P.D., Smith, D.T. \& Stanford, P.N., Resistivity porosity particle shape relationships for marine sands. Geophysics, 43: 1250-1268, 1978.

[18] Jaynes, D.B., Colvin, T.S. \& Ambuel, J., Yield mapping by electromagnetic induction. In P.C. Robert et al. (ed.) Proc. of site-specific management for agricultural systems, 2nd, Minneapolis, MN. 27-30 Mar. 
1994. University of Minnesota Extension Service, Minneapolis, MN. 383394, 1995.

[19] Keller, G.V., Effect of wettability on the electrical resistivity of sand. Oil and Gas Journal, 51: 62-65, 1953.

[20] Kelly, B.F.J., Electrical Properties of Sediments and the Geophysical Detection of Groundwater Contamination, The University of New South Wales Sydney, 1-110 pp, 1994.

[21] Lindsay, W.L., Chemical equilibria in soils, John Wiley and Sons, New York, 449, 1979.

[22] Lockhart, N.C., Electrical properties of the surface characteristics and structure of clays: I. Swelling clays. Colloid Interface Sci, 74: 509-529, 1980.

[23] Lund, E.D., Christy, C.D. \& Drummond, P.E., Practical applications of soil electrical conductivity mapping. Veris Technologies, $601 \mathrm{~N}$. Broadway, Salina Kansas 67401, USA, 1999.

[24] McNeal, B.L., Oster, J.D., Hatcher, J.T., Calculation of electrical conductivity from solution composition data as an aid to in-situ estimation of soil salinity. Soil Science, 110: 405-414, 1970.

[25] Nadler, A. \& Frenkel, H., Determination of Soil Solution Electrical Conductivity from Bulk Soil Electrical Conductivity Measurements by the Four-Electrode Method Soil Science Society of America, 44: 1216 1221, 1980.

[26] Patnode, H.W. \& Wyllie, R.J., The presence of conductive solids in reservoir rocks as a factor in Electric log interpretation. Trans. AIME (Pet.) 189: 47-52, 1950.

[27] Pfannkuch, H.O., On the Correlation of Electrical Conductivity Properties of Porous Systems with Viscous Flow Transport Coefficients in Fundamentals of Transport Phenomena in Porous Media, Elsevier Publishing, New York: 42-54, 1972.

[28] Quirk, J.P., Interparticle forces: a basis for the interpretation of soil physical behaviour. Advances in Agronomy 53: 121-177, 1994.

[29] Revil, A. \& Glover, P., Nature of surface electrical conductivity in natural sands, sandstones, and clays Geophysical Research Letters. 25(5): 691694, 1998.

[30] Rhoades, J.D. and Ingvalson, R. D., Determining salinity in field soils with soil resistance measurements. Soil Science Society of America, 35: 54-60, 1971.

[31] Rhoades, J.D., Manteghi, N.A., Shouse, P.J. and Alves, W.J., Soil electrical conductivity and soil salinity: new formulations and calibrations. Soil Science Society of America, 53: 433-439, 1989.

[32] Rhoades, J.D., Ingvalson, R.D. \& Hatcher, J.T., Laboratory determination of leachable soil boron. Soil Science Society of America, 34: 871-875, 1970.

[33] Rhoades, J.D. \& Van Schilfgaarde, J., An Electrical Conductivity Probe for Determining Soil Salinity. Soil Science Society of America 40: 647$651,1976$. 
[34] Schmitz, R.M., Can the diffuse double layer theory describe changes in hydraulic conductivity of compacted clays? Geotechnical and Geological Engineering, 24: 1835-1844, 2006.

[35] Silva, P.L. \& Bassiouni, Z., Hydrocarbon Saturation Equation in Shaly Sands According to the S-B Conductivity Model, SPE Formation Evaluation, September Issue, 503-509, 1988.

[36] Smith-Rose, R.L., The electrical properties of soil for alternating currents at radio frequencies. Royal Society Publishing, London, Ser. A 140:359 $377,1933$.

[37] Stewart, W.M. \& Hossner, L.R., Ecological Risk Assessment Factors Affecting the Ratio of Cation Exchange Capacity to Clay Content in Lignite Overburden. Environmental Quality, 30: 1143-1149, 2001.

[38] Taylor, S., and Barker, R. Modelling the DC electrical response of fully and partially saturated Permo-Triassic sandstone. Geophysical Prospecting, 54: 351-367, 2005

[39] Toumelin, E. \& Torres-Verdin, C., Influence of Oil Saturation and Wattability on Rocks Resistivity Measurements: A Uniform Pore-scale Approach, SPWLA 46 Annual Logging Symposium, June 26-29, 2005.

[40] U.S. Salinity Laboratory Staff, Diagnosis and improvement of saline and alkali soils, USDA Handbook 60. U.S. Government Printing Office, Washington, DC, USA, pp. 1-160, 1954.

[41] Vezenov, D.V., Aleksandr Noy, Rozsnyai., L.F \& Lieber, C.M., Force Titrations and Ionization State Sensitive Imaging of Functional Groups in Aqueous Solutions by Chemical Force Microscopy. Journal of the American Chemical Society, 119: 2006-20015, 1997.

[42] Waxman, M.H. \& Smits, L.J.M., Electrical Conductivities in Oil Bearing Shaly Sands. Soc. of Petroleum Engineers, Proc. of 42nd Annual Fall Mtg., Houston, Tx., SPE-1863-A, pp. V-145-V-160, 1968.

[43] Wenner, F., A method of measuring earth-resistivity. United States Bureau of Standards Science. (258), 1915.

[44] Winsauer, W.O., Shearin, H.M., Masson, P.H. \& Williams, M., Resistivity of brine-saturated sands in relation to pore geometry American Association of Petroleum Geology Bulletin 36: 253-277, 1952.

[45] Winsauer,W.O. \& McCardell,W.M., Ionic double-layer conductivity in reservoir rocks: Trans. Am. Inst. Min., Metall., 198,129-134, 1953.

[46] Worthington, P.E., The evolution of shaly sand concepts in reservoir evaluation, The Log Analyst. 26, 1(23-40), 1985.

[47] Worthington, P.F. \& Pallat, N., Effect of Variable Saturation Exponent upon the Evaluation of Hydrocarbon Saturation. SPE paper 20538 101110,1989 